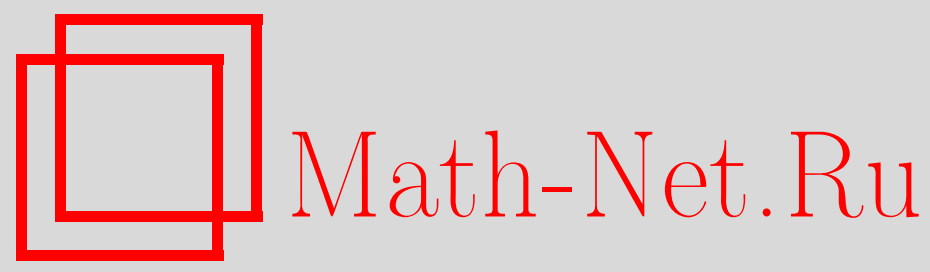

О. П. Филатов, Существование пределов максимальных средних, Матем. заметки, 2000, том 67, выпуск 3, 433-440

DOI: https://doi.org/10.4213/mzm855

Использование Общероссийского математического портала Math-Net.Ru подразумевает, что вы прочитали и согласны с пользовательским соглашением http://www . mathnet.ru/rus/agreement

Параметры загрузки:

IP: 35.173 .219 .12

26 апреля 2023 г., 18:26:45

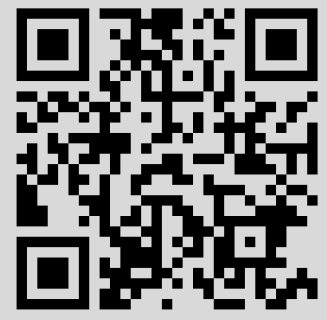




\section{СУЩЕСТВОВАНИЕ ПРЕДЕЛОВ МАКСИМАЛЬНЫХ СРЕДНИХ}

\section{О.П. Филатов}

Для класса $\Pi\left(\mathbb{R}^{m}\right)$ непрерывных почти периодических функций $f: \mathbb{R}^{m} \rightarrow \mathbb{R}$ рассматривается задача о существовании предела

$$
M_{f}=\lim _{\Delta \rightarrow \infty} \sup _{\gamma} \frac{1}{\Delta} \int_{0}^{\Delta} f(\gamma(t)) d t,
$$

где точная верхняя грань берется по всем решениям в смысле Каратеодори дифференциального включения $\dot{\gamma} \in G, \gamma(0)=a_{0}$. Установлено, что если компакт $G \subset \mathbb{R}^{m}$ не содержится в подпространстве из $\mathbb{R}^{m}$ размерности $m-1$ (т.е. является невырожденньмм), то предел существует равномерно по начальному вектору $a_{0} \in \mathbb{R}^{m}$. Обратно, если предел существует для любой функции $f \in \Pi\left(\mathbb{R}^{m}\right)$ равномерно по начальному вектору $a_{0} \in \mathbb{R}^{m}$, то компакт $G$ будет невырожденным. Доказано также существование экстремального решения, для которого реализуется равномерный по начальным условиям предел максимального среднего.

Библиографйл: 5 названий.

1. Постановка задачи. Пусть непрерьвная функция $f: \mathbb{R}^{m} \rightarrow \mathbb{R}$ является почти периодической в евклидовом пространстве $\mathbb{R}^{m}$ со скалярным произведением $\langle\cdot, \cdot\rangle$. Множество всех таких функций будем обозначать через $\Pi\left(\mathbb{R}^{m}\right)$. Рассмотрим задачу о существовании предела

$$
M_{f}=\lim _{\Delta \rightarrow \infty} \sup _{\gamma} \frac{1}{\Delta} \int_{0}^{\Delta} f(\gamma(t)) d t,
$$

где точная верхняя грань вычисляется по всем решениям в смысле Каратеодори дифференциального включения

$$
\dot{\gamma} \in G, \quad \gamma(0)=a_{0} .
$$

Здесь $G$ - компактное множество из $\mathbb{R}^{m}$ (мы отождествляем фазовое и касательное пространства). Множество всех решений задачи (2), определенных в промежутке $t \geqslant 0$, обозначаем через $\Gamma\left(a_{0}\right)$.

В [1] было показано, что для почти периодической функции $f$ одной скалярной переменной $(m=1)$ предел $(1)$ в случае, когда компакт $G \neq\{0\}$ является отрезком, сушествует равномерно по начальному условию $a_{0} \in \mathbb{R}$.

Работа выполнена при финансовой поддержке Российского фонда фундаментальных исследований, грант № 96-01-00616. 
Здесь ставится следующая задача: в многомерном случае $m \geqslant 2$ указать свойства компакта $G$, которые, с одной стороны, являются достаточньми условиями существования пределов максимальных средних равномерно по начальным условиям $a_{0} \in \mathbb{R}^{m}$ для любой данной функции из класса $\Pi\left(\mathbb{R}^{m}\right)$, а с другой стороны, являются необходимьми условиями сразу для всего класса $\Pi\left(\mathbb{R}^{m}\right)$ в том смысле, что если для всех $f \in \Pi\left(\mathbb{R}^{m}\right)$ предел (1) существует равномерно по начальным условиям для дифференциального включения (2), то компакт $G$ обязан удовлетворять этим свойствам.

Кроме того, дается ответ на естественньй вопрос о существовании экстремального решения дифференциального включения (2), для которого реализуется соответствующий предел (1). Последняя задача решается в более общей постановке, чем первая.

Отметим, что условие равномерности является одним из основных требований в теоремах усреднения дифференциальных включений с быстрьми и медленными переменными [2].

2. Основные определения. Для непрерывной функции $f: \mathbb{R}^{m} \rightarrow \mathbb{R}$ мы используем наиболее подходящее для рассматриваемой задачи определение почти периодичности по Г. Бору: для любого $\varepsilon>0$ найдется $l=l(\varepsilon)>0$ такое, что любой $m$-мерный куб пространства $\mathbb{R}^{m}$ с длинами сторон равньми $l$ содержит векторный $\varepsilon$-почти период $T=T(\varepsilon)$ функции $f$, т.е. для любого $\gamma \in \mathbb{R}^{m}$ вьполняется неравенство $|f(\gamma+T)-f(\gamma)|<\varepsilon$.

Заметим, что почти периодическая функция является ограниченной:

$$
\sup _{x \in \mathbb{R}^{m}}|f(x)|=K<\infty
$$

Компакт $G \subset \mathbb{R}^{m}$ будем назьвать невырожсденным, если он не принадлежит подпространству $\mathbb{R}^{m}$ размерности $m-1$.

3. Основные результаты. Следующая теорема обобщает известное свойство существования среднего для почти периодической функции в одномерном случае. В приведенной вьше постановке задачи это соответствует ситуации $m=1$, при этом компакт $G$ дифференциального включения (2) совпадает с точкой, отличной от нуля.

ТЕорема 1. Пусть компакт $G$ является невырохсенным. Тогда для любой почти периодической функиии $f \in \Pi\left(\mathbb{R}^{m}\right)$ предел максимального среднего (1) существует равномерно по начальному вектору $a_{0} \in \mathbb{R}^{m}$.

Обратно, если для данного дифференциального включения (2) предел максимального среднего (1) существует равномерно по начальному вектору $a_{0} \in \mathbb{R}^{m}$ для любой функиии $f \in \Pi\left(\mathbb{R}^{m}\right)$, то компакт $G$ является невырожсденным.

В следуюшей теореме решается вопрос о существовании экстремального решения для предела (1), где точная верхняя граница берется по всем решениям дифференциального включения

$$
\dot{\gamma} \in G(\gamma), \quad \gamma(0)=a_{0}
$$

при этом далее предполагается, что многозначное отображение $G$ из $\mathbb{R}^{m}$ в совокупность множеств из пространства $\mathbb{R}^{m}$ таково, что любое решение дифференциального включения (3) определено при любом начальном векторе $a_{0} \in \mathbb{R}^{m}$ для всех $t \geqslant 0$. 
Теорема 2. Пусть отображение $f: \mathbb{R}^{m} \rightarrow \mathbb{R}$ локально интегрируемо по Лебегу на любом решении дифференииального включения (3), равномерно ограничено $(|f| \leqslant K<\infty)$ и существует предел максимального среднего (1) равномерно по начальному вектору $a_{0} \in \mathbb{R}^{m}$. Тогда существует решение $\gamma(t)$ задачи (3), для которого достигается предел максимального среднего

$$
M_{f}=\lim _{\Delta \rightarrow \infty} \frac{1}{\Delta} \int_{0}^{\Delta} f(\gamma(t)) d t .
$$

Таким образом, из существования предела максимального среднего следует существование экстремального решения при весьма общих требованиях на функцию $f$ и дифференциальное включение. В частности, в условиях теоремы 1 для почти периодической функции $f: \mathbb{R}^{m} \rightarrow \mathbb{R}$ всегда сушествует такое решение.

\section{4. Доказательство теорем.}

ДокАЗАТЕЛЬСтво тЕОРЕмЫ 1. Сначала будем считать, что компакт $G$ является выпукльм множеством. Тогда множество $G$ совпадает с его выпуклой оболочкой $\operatorname{co}(G)$. Предположим, что компакт $G$ является невырожденным. Если размерность компакта $\operatorname{dim}(G)=m-1$ (это размерность его аффинной оболочки), то множество $G$ содержится в некоторой гиперплоскости $\Gamma_{m}$, определяемой уравнением $\left\langle e_{m}, \xi\right\rangle=b$ относительно вектора скорости $\xi=\dot{\gamma} \in \mathbb{R}^{m}$, где $e_{m}$ - единичньй нормальный вектор гиперплоскости, число $b \neq 0$. Тогда по известной теореме о непустоте относительной внутренности выпуклого множества [3, теорема 1.5, с. 202$]$ получаем, что множество $G$ содержит пересечение гиперплоскости $\Gamma_{m}$ с некоторым шаром пространства $\mathbb{R}^{m}$. В таком случае задачу $(1),(2)$ запишем в координатах $\left(\gamma_{1}, \gamma_{2}, \ldots, \gamma_{m}\right)$ относительно базиса $e_{1}, e_{2}, \ldots, e_{m}$, где первые $m-1$ векторов образуют базис в линейном подпространстве, ассоциированном с гиперплоскостью $\Gamma_{m}$. Из построения следует, что компакт $G$ содержит множество $P=\sum_{j=1}^{m} \xi_{j} e_{j}$, где координатный вектор $\left(\xi_{1}, \xi_{2}, \ldots, \xi_{m}\right)$ скорости $\xi$ принадлежит промежутку вида

$$
P_{\xi}=\left[\omega_{1}^{1}, \omega_{2}^{1}\right] \times \cdots \times\left[\omega_{1}^{m}, \omega_{2}^{m}\right] ;
$$

здесь $\omega_{1}^{j}, \omega_{2}^{j}$-постоянные, при этом $\omega_{2}^{j}-\omega_{1}^{j}>0$ для всех $j=1,2, \ldots, m-1, \omega_{1}^{m}=\omega_{2}^{m}$. Последнее равенство в случае $\operatorname{dim}(G)=m$ заменяется на строгое неравенство $\omega_{1}^{m}<\omega_{2}^{m}$, при этом выбор базиса пространства $\mathbb{R}^{m}$ произволен. Таким образом, в общем случае, когда компакт $G$ является невырожденным, промежуток (5) является прямым произведением первых $m-1$ отрезков положительной длины и отрезка, которьй, возможно, содержит единственную точку отличную от нуля. В любом случае существует число $\omega_{m} \neq 0, \omega_{m} \in\left[\omega_{1}^{m}, \omega_{2}^{m}\right]$. Дальнейшие рассуждения проводятся в указанных координатах. Для простоты мы будем считать, что исходная задача записана сразу в требуемых координатах, при этом мы полагаем $\gamma=\left(\gamma_{1}, \gamma_{2}, \ldots, \gamma_{m}\right), \xi=\left(\xi_{1}, \xi_{2}, \ldots, \xi_{m}\right)$.

Пусть теперь

$$
J_{\Delta}\left(\gamma_{0}\right)=\bigcup_{\gamma} I_{\Delta}\left(\gamma_{0}, \gamma\right), \quad I_{\Delta}\left(\gamma_{0}, \gamma\right)=\frac{1}{\Delta} \int_{0}^{\Delta} f(\gamma(t)) d t,
$$

где объединение средних берется по всем решениям $\gamma \in \Gamma\left(\gamma_{0}\right)$ для некоторого начального вектора $\gamma_{0} \in \mathbb{R}^{m}$. Покажем, что утверждение теоремы является следствием теоремы 1 из [4] о существовании усредненного дифференциального включения. В указанной теореме достаточно проверить условие асимптотической независимости средних 
$I_{\Delta}\left(\gamma_{0}, \gamma\right)$ от начального вектора $\gamma_{0} \in \mathbb{R}^{m}$ (поскольку дифференциальное включение $(2)$ является автономньм). Это условие означает, что

$$
\begin{gathered}
\forall \varepsilon>0 \quad \exists \Delta(\varepsilon)>0 \quad \forall \gamma_{01}, \gamma_{02} \in \mathbb{R}^{m} \quad \forall \gamma_{1} \in \Gamma\left(\gamma_{01}\right) \quad \exists \gamma_{2} \in \Gamma\left(\gamma_{02}\right): \\
\left|I_{\Delta}\left(\gamma_{01}, \gamma_{1}\right)-I_{\Delta}\left(\gamma_{02}, \gamma_{2}\right)\right| \leqslant \varepsilon
\end{gathered}
$$

при всех $\Delta \geqslant \Delta(\varepsilon)$.

Поскольку функция $f$ непрерывна, асимптотическая независимость средних от начального вектора согласно [4, теорема 1] гарантирует существование отрезка $\left[m_{1}, m_{2}\right]$, для которого

$$
\lim _{\Delta \rightarrow \infty} \alpha\left(J_{\Delta}\left(\gamma_{0}\right),\left[m_{1}, m_{2}\right]\right)=0
$$

равномерно по $\gamma_{0} \in \mathbb{R}^{m}$, где $\alpha(\cdot, \cdot)$ - отклонение по Хаусдорфу для множеств из $\mathbb{R}$. Поскольку в этом случае предел максимального среднего (1) совпадает с числом $m_{2}$, достаточно проверить условие асимптотической независимости средних от начального вектора $\gamma_{0} \in \mathbb{R}^{m}$.

Пусть $\gamma_{01}=\left(a_{1}, a_{2}, \ldots, a_{m}\right), \gamma_{02}=\left(b_{1}, b_{2}, \ldots, b_{m}\right)$. Покажем сначала, что можно выбрать число $t_{1}>0$ и числа $\omega_{j} \in\left[\omega_{1}^{j}, \omega_{2}^{j}\right], j=1,2, \ldots, m$, так, чтобы вьполнялись равенства

$$
b_{j}+t_{1} \omega_{j}=a_{j}+T_{j}
$$

для некоторого векторного $\varepsilon / 2$-почти периода $T=\left(T_{1}, T_{2}, \ldots, T_{m}\right)$ функции $f$. Положим $c_{j}=a_{j}-b_{j}$. Последнее уравнение $(j=m)$ системы $(6)$ преобразуем к виду

$$
t_{1}=\frac{c_{m}+T_{m}}{\omega_{m}} .
$$

Условия $\omega_{j} \in\left[\omega_{1}^{j}, \omega_{2}^{j}\right]$ при $t_{1}>0$ согласно (6) запишем в виде эквивалентной системы неравенств

$$
t_{1} \omega_{1}^{j}-c_{j} \leqslant T_{j} \leqslant t_{1} \omega_{2}^{j}-c_{j}, \quad j=1,2, \ldots, m-1 .
$$

Здесь число $T_{j}$ принадлежит отрезку, длина которого равна $t_{1}\left(\omega_{2}^{j}-\omega_{1}^{j}\right)$. Поэтому пусть

$$
\delta=\min _{1 \leqslant j \leqslant m-1}\left\{\omega_{2}^{j}-\omega_{1}^{j}\right\}, \quad \rho=\max _{1 \leqslant j \leqslant m-1}\left\{\left|\omega_{1}^{j}\right|\right\} .
$$

Выберем число $t_{1, \min }$ так, чтобы $t_{1, \min } \delta / 2 \geqslant l$. Кроме того, потребуем, чтобы

$$
\frac{2 l \rho}{t_{1, \min }\left|\omega_{m}\right|} \leqslant \frac{\delta}{2} \text {. }
$$

Следовательно, можно принять

$$
t_{1, \min }=\max \left\{\frac{2 l}{\delta}, \frac{4 l \rho}{\left|\omega_{m}\right| \delta}\right\} .
$$

Кроме того, пусть $t_{2, \max }=t_{1, \min }+2 l /\left|\omega_{m}\right|$.

Рассмотрим теперь семейство $m$-мерньх промежутков

$$
P(k)=\left[d_{1}^{1}, d_{2}^{1}\right] \times \cdots \times\left[d_{1}^{m-1}, d_{2}^{m-1}\right] \times[k l,(k+1) l],
$$


где $d_{i}^{j}=\tau_{i}^{j} \omega_{i}^{j}-c_{j}, i=1,2, j=1,2, \ldots, m-1, k=0, \pm 1, \pm 2, \ldots$, a постоянные $\tau_{1}^{j}, \tau_{2}^{j}$ определяются формулами

$$
\tau_{1}^{j}=\left\{\begin{array}{ll}
t_{2, \max }, & \text { если } \omega_{1}^{j}>0, \\
t_{1, \min }, & \text { если } \omega_{1}^{j} \leqslant 0,
\end{array} \quad \tau_{2}^{j}= \begin{cases}t_{1, \min }, & \text { если } \omega_{2}^{j}>0, \\
t_{2, \max }, & \text { если } \omega_{2}^{j} \leqslant 0 .\end{cases}\right.
$$

Так как $d_{2}^{j}-d_{1}^{j}=\tau_{2}^{j}\left(\omega_{2}^{j}-\omega_{1}^{j}+\left(1-\tau_{1}^{j} / \tau_{2}^{j}\right) \omega_{1}^{j}\right)$, то $d_{2}^{j}-d_{1}^{j} \geqslant t_{1, \min }(\delta-\delta / 2)=l$. Следовательно, для любого целого $k$ существует векторный $\varepsilon / 2$-почти период

$$
T=T\left(k, \frac{\varepsilon}{2}\right)=\left(T_{1, k}, T_{2, k}, \ldots, T_{m, k}\right) \in P(k) .
$$

В частности, выполняются неравенства $k l \leqslant T_{m, k} \leqslant(k+1) l$, которые в случае $\omega_{m}>0$ эквивалентны соотношениям

$$
d_{m, k} \leqslant \frac{c_{m}+T_{m, k}}{\omega_{m}} \leqslant d_{m, k+1}
$$

где $d_{m, k}=\left(c_{m}+k l\right) / \omega_{m}$.

В один из отрезков $\left[d_{m, k}, d_{m, k+1}\right]$ попадет и число $t_{1, \min }$. Соответствующее значение $k$ обозначим через $s$. В таком случае, число $t_{1, \min } \in\left[d_{m, s}, d_{m, s+1}\right]$. Положим $T_{j}=T_{j, s+1}$ для любого $j$ и по формуле (7) вычислим $t_{1}$. Так как $d_{m, s+1} \leqslant t_{1} \leqslant d_{m, s+2}$, то $0 \leqslant t_{1}-t_{1, \min } \leqslant d_{m, s+2}-d_{m, s}=2 l / \omega_{m}$.

Если $\omega_{m}<0$, то аналогично получим соотношения $d_{m, s+1} \leqslant\left(c_{m}+T_{m, s}\right) / \omega_{m} \leqslant d_{m, s}$, при этом в указанном отрезке находится и число $t_{1, \min }$. Поэтому принимаем $T_{j}=T_{j, s-1}$ для любого $j$ и определяем $t_{1}$ по формуле $(7)$. В этом случае

$$
0 \leqslant t_{1}-t_{1, \min } \leqslant d_{m, s-1}-d_{m, s+1}=-\frac{2 l}{\omega_{m}}=\frac{2 l}{\left|\omega_{m}\right|} .
$$

Таким образом, если $\omega_{m} \neq 0$, то всегда $t_{1, \min } \leqslant t_{1} \leqslant t_{1, \max }$. Следовательно, если теперь определить числа $\omega_{j}=\left(T_{j}+c_{j}\right) / t_{1}, j=1,2, \ldots, m-1$, то $\omega_{j} \in\left[\omega_{1}^{j}, \omega_{2}^{j}\right]$, так как $\left(T_{j}+c_{j}\right) / t_{1} \geqslant \tau_{1}^{j} \omega_{1}^{j} / t_{1} \geqslant \omega_{1}^{j}$, а с другой стороны, $\left(T_{j}+c_{j}\right) / t_{1} \leqslant \tau_{2}^{j} \omega_{2}^{j} / t_{1} \leqslant \omega_{2}^{j}$.

Таким образом, $\omega=\left(\omega_{1}, \omega_{2}, \ldots, \omega_{m}\right) \in P_{\xi}$, удовлетворяется система неравенств (8), а значит и система (6), при этом имеют место соотношения

$$
t_{1, \min } \leqslant t_{1} \leqslant t_{1, \max }
$$

где постоянные $t_{1, \min }, t_{1, \max }$ не зависят от начального вектора.

Возьмем теперь произвольное решение $\gamma_{1} \in \Gamma\left(\gamma_{01}\right)$ и построим решение $\gamma_{2} \in \Gamma\left(\gamma_{02}\right)$ следующего вида:

$$
\gamma_{2}(t)= \begin{cases}\gamma_{02}+t \omega, & \text { если } 0 \leqslant t \leqslant t_{1}, \\ \gamma_{1}\left(t-t_{1}\right)+T, & \text { если } t \geqslant t_{1},\end{cases}
$$

где $T=\left(T_{1}, T_{2}, \ldots, T_{m}\right)$. Тогда

$$
\gamma_{2}(0)=\gamma_{02}, \quad \gamma_{2}\left(t_{1}\right)=\gamma_{01}+T
$$


Среднее $I_{\Delta}^{2}=I_{\Delta}\left(\gamma_{02}, \gamma_{2}\right)$ представим в виде суммы двух интегралов

$$
\begin{aligned}
I_{\Delta}^{2} & =\frac{1}{\Delta} \int_{0}^{t_{1}} f\left(\gamma_{2}(t)\right) d t+\frac{1}{\Delta} \int_{t_{1}}^{\Delta} f\left(\gamma_{2}(t)\right) d t \\
& =\frac{1}{\Delta} \int_{0}^{t_{1}} f\left(\gamma_{2}(t)\right) d t+\frac{1}{\Delta} \int_{0}^{\Delta-t_{1}} f\left(\gamma_{1}(t)+T\right) d t .
\end{aligned}
$$

Здесь использовалось правило построения решения $\gamma_{2}(t)$. С другой стороны, среднее

$$
I_{\Delta}^{1}=\frac{1}{\Delta} \int_{\Delta-t_{1}}^{\Delta} f\left(\gamma_{1}(t)\right) d t+\frac{1}{\Delta} \int_{0}^{\Delta-t_{1}} f\left(\gamma_{1}(t)\right) d t .
$$

Сравнивая с предыдущим равенством и учитьвая (9), получим оценку

$$
\left|I_{\Delta}^{1}-I_{\Delta}^{2}\right| \leqslant \frac{2 t_{1} K}{\Delta}+\frac{\varepsilon}{2} \leqslant \frac{2 K t_{1, \max }}{\Delta}+\frac{\varepsilon}{2} .
$$

Следовательно, в определении асимптотической независимости средних от начальных условий можно положить $\Delta(\varepsilon)=4 K t_{1, \max } / \varepsilon$, что в условиях теоремы и доказьвает сушествование предела максимального среднего.

Таким образом, для выпуклого невырожденного компакта $G \subset \mathbb{R}^{m}$ всегда существует предел максимального среднего для любой функции $f \in \Pi\left(\mathbb{R}^{m}\right)$.

Если множество $G$ не является выпуклым, то воспользуемся известной связью между решениями задачи (2) и решениями дифференциального включения $\dot{\gamma} \in \operatorname{co}(G)$ с теми же начальньми условиями. Именно, на любом конечном отрезке любое решение последнего дифференциального включения можно аппроксимировать в равномерной метрике с наперед заданной точностью некоторым решением задачи (2) (см., например, [5, теорема 10, с. 219]).

Следовательно, асимптотическая независимость средних от начального вектора $a_{0} \in \mathbb{R}^{m}$ имеет место и для невьпуклого невырожденного компакта $G \subset \mathbb{R}^{m}$.

Заметим, что формально теорема 1 из [4], на основании которой было доказано сушествование максимального среднего для выпуклого компакта $G$, сформулирована для дифференциальных включений с вьпуклозначными правыми частями. Однако в доказательстве самой теоремы использовались только свойства непрерьвности функции $f$ (в [4, теорема 1] это многозначное отображение) и асимптотической независимости средних от начальных данных при условии, что все решения дифференциального включения определены в бесконечном промежутке $t \geqslant t_{0}$ при любых начальных условиях $t_{0} \geqslant 0$, $a_{0} \in \mathbb{R}^{m}$. Поскольку в нашем случае эти требования выполняются, ссылка на указанную теорему корректна, что и завершает доказательство первой части теоремы.

Допустим теперь, что в классе функций $\Pi\left(\mathbb{R}^{m}\right)$ предел максимального среднего (1) существует равномерно по начальным условиям задачи (2), и предположим, что компакт $G$ является вырожденным. Следовательно, множество $G$ содержится в гиперплоскости $\Gamma_{m}$, проходящей через нуль пространства $\mathbb{R}^{m}$. Тогда если ввести координаты $\gamma$ точно так же, как и при доказательстве достаточности, то для функции $f(\gamma)=\sin \gamma_{m}$, заданной в этих координатах, предел максимального среднего (1) равен $\sin \gamma_{m}(0)$ (так как $\dot{\gamma}_{m}=0$ ) и поэтому зависит от начального условия. Теорема 1 доказана.

Отметим, что для любого компакта $G \subset \mathbb{R}^{m}$ всегда можно указать функцию $f \in \Pi\left(\mathbb{R}^{m}\right)$ такую, что предел максимального среднего (1) для нее существует равномерно по начальному вектору $a_{0} \in \mathbb{R}^{m} ;$ при этом если компакт $G \neq\{0\}$, то такая функция $f$ отлична от постоянной. 
ДОКАЗАТЕЛЬСТВО ТЕОРЕМЫ 2. Из существования предела максимального среднего (1) равномерно по начальным условиям следует, что для любого начального условия $\left(t_{0}, \gamma_{0}\right) \in \mathbb{R} \times \mathbb{R}^{m}$ и любого числа $\varepsilon_{k}=1 / k$ существует $\Delta_{k}>0$, не зависящее от выбора начального условия, такое, что на любом отрезке $\left[t_{0}, t_{0}+\Delta_{k}\right]$ найдется решение $\gamma_{k}(t)$ дифференциального включения $(3), \gamma\left(t_{0}\right)=\gamma_{0}$, для которого вьполняется соотношение

$$
\frac{1}{\Delta_{k}} \int_{t_{0}}^{t_{0}+\Delta_{k}} f\left(\gamma_{k}(t)\right) d t=M_{f}+\varphi_{k}
$$

где $\left|\varphi_{k}\right|<\varepsilon_{k}$. При этом можно считать, что последовательность $\left\{\Delta_{k}\right\}$ является неубывающей.

Решение $\gamma(t)$ строится последовательно на отрезках длины $\Delta_{k}$ с использованием решений вида (10) для соответствующих начальных условий, при этом отрезок длины $\Delta_{k}$ откладывается вправо $n_{k}$ раз, где целые $n_{k}$ образуют также неубывающую последовательность и выбираются так, чтобы при $k \geqslant 2$ выполнялись неравенства

$$
\frac{n_{k-1}}{n_{k}}<2^{-k}, \quad \frac{\Delta_{k+1} K_{1}}{n_{1}+n_{2} \Delta_{2}+\cdots+n_{k} \Delta_{k}}<\varepsilon_{k+1},
$$

где постоянная $K_{1}=\max \{1, K\}$.

На первом из отрезков $\left[0, \Delta_{1}\right]$ функция $\gamma(t)$ совпадает с решением $\gamma_{1}(t), \gamma_{1}(0)=a_{0}$, для которого вьполняется соотношение $(10)$. В общем случае на $k$-й серии последовательных отрезков (их число равно $n_{k}$ ) каждое последуюшее решение непрерьвно продолжает предыдущее и удовлетворяет равенству вида (10). В результате решение $\gamma(t)$ оказьвается построенным на всей полуоси $[0, \infty)$.

Положим теперь $\Delta_{k-1, j}=n_{1} \Delta_{1}+n_{2} \Delta_{2}+\cdots+n_{k-1} \Delta_{k-1}+j \Delta_{k}$, где $0 \leqslant j \leqslant n_{k}$, $k \geqslant 2$. На отрезке $\left[0, \Delta_{k-1, j}\right]$ среднее представим в виде

$$
\frac{1}{\Delta_{k-1, j}} \int_{0}^{\Delta_{k-1, j}} f(\gamma(t)) d t=\frac{1}{\Delta_{k-1, j}}\left(\sum_{i=1}^{k-1} \sum_{s=1}^{n_{i}} \Delta_{i}\left(M_{f}+\varphi_{i, s}\right)+\sum_{s=1}^{j} \Delta_{k}\left(M_{f}+\varphi_{k, s}\right)\right)
$$

где каждая из функций $\varphi_{i, s}$ допускает оценку $\left|\varphi_{i, s}\right|<\varepsilon_{i}$, как это следует из правила построения решения $\gamma(t)$. Отсюда

$$
\frac{1}{\Delta_{k-1, j}} \int_{0}^{\Delta_{k-1, j}} f(\gamma(t)) d t=M_{f}+\eta_{k-1, j}
$$

где для функции $\eta_{k-1, j}$ получим оценку

$$
\left|\eta_{k-1, j}\right| \leqslant \frac{j \Delta_{k} \varepsilon_{k}}{\Delta_{k-1, j}}+\sum_{i=1}^{k-1} \frac{n_{i} \Delta_{i} \varepsilon_{i}}{\Delta_{k-1, j}}
$$

Согласно (11) правая часть последнего неравенства не превосходит величины $1 / k+$ $(k-2) / 2^{k-1}+1 /(k-1)$, поэтому заведомо выполняется соотношение

$$
\left|\eta_{k-1, j}\right|<\frac{k-2}{2^{k-1}}+\frac{2}{k-1}
$$


Если теперь $\Delta_{k-1, j} \leqslant \Delta \leqslant \Delta_{k-1, j}+\Delta_{k}$, то среднее представим в виде

$$
\begin{aligned}
\frac{1}{\Delta} \int_{0}^{\Delta} f(\gamma(t)) d t= & \frac{1}{\Delta_{k-1, j}} \int_{0}^{\Delta_{k-1, j}} f(\gamma(t)) d t+\left(\frac{1}{\Delta}-\frac{1}{\Delta_{k-1, j}}\right) \int_{0}^{\Delta_{k-1, j}} f(\gamma(t)) d t \\
& +\frac{1}{\Delta} \int_{\Delta_{k-1, j}}^{\Delta} f(\gamma(t)) d t .
\end{aligned}
$$

Отсюда на основании (12), (13) следует

$$
\frac{1}{\Delta} \int_{0}^{\Delta} f(\gamma(t)) d t=M_{f}+\sigma(\Delta)
$$

где

$$
|\sigma(\Delta)| \leqslant\left|\eta_{k-1, j}\right|+2 \varepsilon_{k} \leqslant \frac{k-2}{2^{k-1}}+\frac{2}{k-1}+\frac{2}{k}
$$

Следовательно,

$$
\lim _{\Delta \rightarrow \infty} \sigma(\Delta)=0
$$

Это и означает, что для построенного решения $\gamma(t)$ выполняется соотношение $(4)$, что и требовалось.

Теорема 2 доказана.

\section{СПИСОК ЦИТИРОВАННОЙ ЛИТЕРАТУРЫ}

[1] Филатов О.П. Вычисление пределов максимальных средних // Матем. заметки. 1996. T. 59. № 5. C. 759-767.

[2] Филатов О.П., Хапаев М. М. Усреднение систем дифференциальных включений. М.: Изд-во МГУ, 1998.

[3] Васильев Ф. П. Численные методы решения экстремальных задач. М.: Наука, 1980.

[4] Филатов О.П. О сушествовании усредненного дифференциального включения // Дифференц. уравнения. 1989. Т. 25. №12. С. 2118-2127.

[5] Благодатских В.И., Филиппов А. Ф. Дифференциальные включения и оптимальное управление // Тр. МИАН. 1985. Т. 169. С. 194-252.

Самарский государственный университет

Поступило

E-mail: filt@ssu.samara.ru

30.07.1997

Исправленный вариант

29.01.1999 